Instituto Internacional de Investigación y Desarrollo Tecnológico Educativo INDTEC, C.A.

DOI: https://doi.org/10.29394/Scientific.issn.2542-2987.2020.5.15.9.191-208

OAI-PMH: http://www.indteca.com/ojs/index.php/Revista Scientific/oai

Artículo Original / Original Article

\title{
La formación docente bajo la modalidad pareja pedagógica practicante en la Universidad Nacional de Educación
}

\author{
Autores: Juan Gabriel Jaramillo Jimbo \\ Universidad Nacional de Educación, UNAE \\ jaramillo271296@gmail.com \\ Azogues, Ecuador \\ https://orcid.org/0000-0001-9241-5411 \\ Justina Elena Orovio Quintero \\ Universidad Nacional de Educación, UNAE \\ ¿ ecuador@hotmail.es \\ Azogues, Ecuador \\ https://orcid.org/0000-0002-2641-7491
}

\section{Resumen}

Este artículo trata sobre la pareja pedagógica practicante, modalidad de formación docente en la Universidad Nacional de Educación (UNAE), fundamentada desde el Modelo Pedagógico y Modelo de Práctica Preprofesional, documentos normativos antecedentes de la investigación. El proceso metodológico parte de la revisión teórico-categórica antes, durante y después del proceso de investigación para asumir el concepto de pareja pedagógica practicante. Así, se entiende como pareja pedagógica practicante a estudiantes que realizan un trabajo cooperativo, reflexivo, de acompañamiento pedagógico en el proceso de la práctica preprofesional. Asimismo, las técnicas e instrumentos útiles en el proceder metodológico involucran a sujetos de la UNAE como de las instituciones donde se realiza la práctica preprofesional. La problemática ¿cuáles serán las funciones de la pareja pedagógica practicante en las unidades de formación?, dirige a la propuesta de cinco funciones (comunicativa, organizativa, ejecutiva, investigativa y evaluativa) con sus respectivos criterios. En efecto, se considera a la pareja pedagógica practicante como una modalidad crucial para fomentar el trabajo cooperativo, diálogo, planificación, retroalimentación, reflexión, entre otros aspectos que se viven cotidianamente en el transcurrir de la práctica.

Palabras clave: formación profesional; docencia; práctica pedagógica.

Cómo citar este artículo:

Jaramillo, J., \& Orovio, J. (2020). La formación docente bajo la modalidad pareja pedagógica practicante en la Universidad Nacional de Educación. Revista Scientific, 5(15), 191-208, e-ISSN: 2542-2987. Recuperado de: https://doi.org/10.29394/Scientific.issn.2542-2987.2020.5.15.9.191-208

Fecha de Recepción: 04-09-2019
Fecha de Aceptación: 23-12-2019
Fecha de Publicación: 05-02-2020 


\title{
Teacher training under the modality pedagogical couple practicing at the National University of Education
}

\begin{abstract}
This article is about the practicing pedagogical couple, teacher training modality at the National University of Education (UNAE), based on the Pedagogical Model and Preprofessional Practice Model, normative documents background of the research. The methodological process starts from the theoretical-categorical review before, during and after the research process to assume the concept of practicing pedagogical couple. Thus, it is understood as a practicing pedagogical couple to students who carry out a cooperative, reflective work, of pedagogical accompaniment in the pre-professional practice process. Likewise, the techniques and instruments useful in the methodological procedure involve subjects of the UNAE as well as the institutions where the pre-professional practice is carried out. The problem, what will be the functions of the pedagogical couple practicing in the training units?, leads to the proposal of five functions (communicative, organizational, executive, investigative and evaluative) with their respective criteria. Indeed, the practicing pedagogical couple is considered as a crucial modality to promote cooperative work, dialogue, planning, feedback, reflection, among other aspects that are lived daily in the course of practice.
\end{abstract}

Keywords: vocational training; teaching profession; teaching practice.

How to cite this article:

Jaramillo, J., \& Orovio, J. (2020). Teacher training under the modality pedagogical couple practicing at the National University of Education. Revista Scientific, 5(15), 191-208, e-ISSN: 2542-2987. Recovered from: https://doi.org/10.29394/Scientific.issn.2542-2987.2020.5.15.9.191-208

Date Received:

04-09-2019
Date Acceptance:

23-12-2019
Date Publication: 05-02-2020 


\section{Introducción}

Una de las experiencias aprendidas desde la práctica preprofesional durante la formación docente bajo la modalidad pareja pedagógica practicante en la Universidad Nacional de Educación (UNAE), es que un solo practicante no alcanza a observar e intervenir en las necesidades o intereses que requiere el aula, considerando que en promedio existen alrededor de 30 niños por aula; mientras que cuatro o cinco practicantes en la misma aula son demasiados; de ahí que se considere la importancia que la formación docente desde la práctica preprofesional se dé bajo la modalidad de pareja pedagógica, en lo que sea posible. En tanto, para que la formación de los estudiantes UNAE tenga éxito desde la práctica preprofesional, es necesario que se disponga de una guía de funciones que orienten el quehacer de al practicante durante su formación.

Desde esa perspectiva, cabe mencionar que la UNAE cuenta con nueve líneas de investigación; dentro de ellas se encuentra el desarrollo profesional docente que abarca las investigaciones relacionadas con la formación inicial de los docentes. Por lo tanto, tal criterio sirve de fundamento para el estudio de la formación docente bajo la modalidad pareja pedagógica practicante. Además, dada la experiencia desde la observación participante como practicantes junto al análisis del Modelo de Práctica Preprofesional, surge la idea de proponer las funciones de la pareja pedagógica practicante que debe cumplir en el proceso de la práctica preprofesional respondiendo a las unidades de formación de la universidad.

Es importante mencionar que uno de los escenarios de la formación profesional docente en la UNAE, es la práctica preprofesional, misma que se da bajo componentes y procesos estipulados en el Modelo de Práctica Preprofesional, según la Universidad Nacional de Educación (UNAE, 2018a): en el cual existe una descripción breve de roles y funciones que también la pareja pedagógica practicante debe cumplir durante su formación, más no una 
organización de funciones según las unidades de formación. Entre los componentes y procesos de la práctica preprofesional se tiene: principios pedagógicos y curriculares (competencias básicas y profesionales), ejes vertebradores, ejes integradores, núcleos problémicos y resultados de aprendizaje de la práctica (pág. 30).

En tanto, el estudio se direcciona desde la problemática ¿Cuáles serán las funciones de la pareja pedagógica practicante en las unidades de formación de la UNAE? Desde ahí se plantea como objetivo general el proponer las funciones de la pareja pedagógica practicante en las unidades de formación a partir de los ejes integradores, núcleos problémicos, competencias básicas y profesionales y, ejes vertebradores, todos ellos como componentes de la práctica preprofesional.

De manera general, la propuesta consiste en definir las funciones que debe cumplir la pareja pedagógica practicante. Las mismas cuentan con tareas específicas propias y comunes en cada unidad que se relacionan con los criterios y acciones que reconocen a la siguiente clasificación de funciones: comunicativa, organizativa, ejecutiva, investigativa y evaluativa. Además, las tareas responden al énfasis del eje vertebrador que se propone considerar en cada unidad de formación. De ahí que se mencione que la propuesta constituye un aporte a la construcción del Modelo de Práctica Preprofesional.

En consecuencia, los siguientes párrafos explican teóricamente la formación profesional docente en la UNAE, el estudio a la pareja pedagógica practicante, el desarrollo de la práctica preprofesional y definiciones de las funciones que se proponen desde este trabajo. Además, desde el aspecto metodológico se describen el análisis de resultados obtenidos en cada una de las etapas que permiten llegar a la elaboración de la propuesta de funciones. $Y$, finalmente se encuentran las conclusiones y recomendaciones que surgen desde la misma investigación en coherencia con el objetivo general antes mencionado. 


\section{Desarrollo (teoría y conceptos, metodología, análisis de resultados y discusión)}

Antes de abordar este apartado, es necesario aclarar que para argumentar el estudio del concepto en sí de pareja pedagógica practicante no se encontraron antecedentes relacionados al propio estudio de dicho concepto. Lo que sí se encontró fueron antecedentes que argumentan sobre práctica preprofesional y de parejas pedagógicas de profesionales. No obstante, los argumentos que exponen los autores consultados sirven de guía para abordar el concepto de pareja pedagógica practicante, puesto que, tales argumentos convergen en un punto común entre la tarea de profesionales y practicantes: la práctica docente.

Dada la explicación, un primer aspecto a abordar es la pareja pedagógica practicante como concepto clave en el desarrollo de la propuesta de funciones. Así, Carniato, Tulián y Velázquez (2015), manifiestan que: "la tarea docente no se realiza en soledad porque es a partir de la mirada del otro que las prácticas pedagógicas cobran sentido y se enriquecen" (pág. 18). A partir de los autores, se puede decir que el trabajo en equipo que se realiza como pareja pedagógica practicante fortalece el acompañamiento pedagógico, diálogo de saberes, el ambiente crítico-reflexivo, responsabilidad común, entre otros aspectos que se viven y desarrollan a diario en la actividad de los practicantes.

Lo manifestado por los autores, no está alejado de lo que significa la pareja pedagógica practicante para la UNAE, puesto que, en su Modelo de Práctica Preprofesional de la Universidad Nacional de Educación (UNAE, 2018b), manifiesta que lo fundamental de estar en pareja para vivir el mundo de la profesión, es entre otros criterios: el "tener la posibilidad de reflexionar acerca de las vivencias en la escuela y de lo que se ha observado en el otro, como parte a la formación de cada uno" (pág. 28); ello con el fin de aportar a la formación de cada uno. 
Por lo tanto, desde los autores se asume que la pareja pedagógica practicante es una modalidad de formación docente en la UNAE que se construye en un ambiente crítico-reflexivo. Funciona como estrategia cooperativa, formativa, de diálogo, acompañamiento pedagógico, experimentación y responsabilidad común. Su actividad ocurre antes, durante y después de la práctica preprofesional en la que se integra con diferentes actores, tanto de la universidad como directivos, docentes, tutores académicos, practicantes y de las instituciones donde se realiza la práctica como estudiantes, tutores profesionales, directivos y representantes legales.

\subsection{Formación profesional docente}

Otra de las categorías abordadas, es la formación profesional docente, Según Porras (2016): ésta se da por dos criterios: uno como práctica de enseñanza donde se desarrolla en sí el proceso formativo y el otro como interiorización del oficio docente en el que se perfecciona y actualiza la práctica de enseñanza (pág. 21). Mientras que, en el Modelo Pedagógico de la Universidad Nacional de Educación (UNAE, 2017a): la formación docente implica el desarrollo de las competencias básicas y profesionales que le permitirán al futuro docente saber hacer, saber pensar, saber sentir, saber investigar (pág. 8). Dicho en otras palabras, la formación no se considera como un ciclo que tiene su principio y fin, sino que es un proceso permanente y presente a diario en los individuos.

En efecto, el docente es un sujeto clave en la educación, pues según Matos, Cegarra y Rivera (2017): él "desempeña un rol de vital importancia en la vida escolar, por cuanto es considerado como el responsable de la docencia" (pág. 323); en tanto que, la responsabilidad se acompaña del compromiso en recibir y apostar por una formación profesional docente permanente. 


\subsection{Práctica preprofesional}

Así mismo, se tiene como categoría lo relacionado a la Práctica Preprofesional, por ser éste el escenario donde también se desenvuelve la pareja pedagógica practicante. En el Modelo Pedagógico de la Universidad Nacional de Educación (UNAE, 2017b): se manifiesta que esta constituye un momento clave para la reflexión y el pensamiento educativo (pág. 22). Mientras, en el Modelo de Práctica Preprofesional de la Universidad Nacional de Educación (UNAE, 2018c): se manifiesta de entre otros criterios, que ésta se sustenta en la teorización de la práctica y la experimentación de la teoría (pág. 34). De acuerdo con la UNAE, la pareja pedagógica practicante teoriza la práctica a partir de la reflexión de las experiencias vividas en la práctica y; experimenta la teoría cuando pone en práctica los conocimientos adquiridos en cada una de las asignaturas del ciclo.

\subsection{Categorías relacionadas con las funciones de la pareja pedagógica practicante}

En cuanto a la comunicación, Brönstrup, Godoi y Ribeiro (2007): manifiestan que comunicar es un encuentro de fronteras perceptivas en el que se intercambian mensajes desde las diferentes perspectivas de cada emisor (pág. 27). Por lo tanto, cada miembro de la pareja pedagógica practicante es un ser cultural diferente, una frontera diferente que se encuentra para dialogar, reflexionar, analizar información, conocimientos, aprendizajes o experiencias. Por otro lado, respecto a la organización, Hayek (1985), citado por López (2005), expone que la organización es: una "ordenación sistemática orientada al logro de determinado fin" (pág. 15). De acuerdo con los autores, para que exista organización es necesario ordenar y estructurar los recursos, que en este caso se considera a los humanos (pareja pedagógica practicante) y materiales (las tareas específicas en cada unidad de formación), para alcanzar el logro de objetivos. 
En la ejecución, Matos y Pasek (2005): manifiestan que ésta es poner en marcha lo planificado como un proceso activo, donde es necesario la motivación, el liderazgo y la comunicación, como elementos para obtener las metas y objetivos propuestos (pág. 103). A decir de los autores, se entiende que la ejecución está estrechamente relacionada con la función comunicativa y organizativa, pues, si hay organización es porque hay comunicación y, por ende, hay ejecución.

Se tiene también como categoría a la investigación, según el Diccionario de la Lengua de la Real Academia Española (RAE, 2001): investigar es un acto de descubrir (pág. 1); y desde la experiencia como practicantes, investigar va más allá del cumplimiento de funciones que permiten descubrir aprendizajes en la formación profesional docente, puesto que al investigar no sólo se aprende, sino que se generan nuevos conocimientos que pueden ser compartidos a un público interesado, específicamente sería un aporte a la comunidad científica. En efecto, cualquier estudiante universitario, está llamado según González, Zerpa, Gutiérrez y Pirela (2007): "a desempeñar un papel clave como investigador de su propia práctica con la finalidad de mejorar su formación" (pág. 280).

Finalmente, se tiene a la evaluación como una función más de la pareja pedagógica practicante. De acuerdo con Mora (2004): ésta puede tener variedad de propósitos, como: "el control y la medición, el enjuiciamiento de la validez del objetivo, la rendición de cuentas" (pág. 2). Siguiendo a los autores, el control y la medición tiene cabida en el momento que se evalúa la asistencia de los practicantes a las escuelas y la rendición de cuentas se evidencia en la reflexión de la práctica. Es necesario recordar a Torres y Torres (2005): quienes presentan la autoevaluación, coevaluación y heteroevaluación, como formas de evaluación que deben desarrollarse "a través del uso de estrategias que consideren no sólo la acumulación de conocimientos, sino también el comportamiento individual y social dentro y fuera de la clase" (pág. 489). 


\section{Metodología y análisis de resultados}

Una vez revisadas teóricamente las categorías afines al objeto de investigación, se presenta a la metodología empleada en el proceso de este estudio. Así pues, la misma atiende a la lógica de la Investigación Acción Participante, bajo el enfoque cualitativo del paradigma sociocrítico. El proceder metodológico se organiza en cuatro etapas, donde el análisis de los resultados se da paulatinamente, a partir de una dimensión (Las funciones de la pareja pedagógica practicante) e indicadores (clasificación de las funciones: comunicativa, organizativa, ejecutiva, investigativa y evaluativa).

\subsection{Primera etapa: diagnóstico inicial}

Durante esta etapa se determinaron aspectos positivos (potencialidades) y aspectos negativos (carencias) encontradas en relación con las funciones de la pareja pedagógica practicante a través de la observación participante y el análisis documental al Modelo de Práctica Preprofesional de la Universidad Nacional de Educación (UNAE).

De manera general, en cuanto a los resultados positivos de la observación participante se observa el reconocimiento de experiencias previas para dialogar y consensuar aspectos de la práctica; la distribución equitativa de tarea; la ayuda y acompañamiento tanto al tutor profesional como estudiantes sea en el diseño de recursos didácticos o en la planificación de unidad didáctica; la socialización de resultados de trabajos investigativos a la comunidad educativa interesada y; la evaluación se desarrolla por la coevaluación, donde implica a la crítica y reflexión. En los resultados negativos, se observa principalmente la escasa orientación a los practicantes sobre las funciones que debe realizar en cada uno de los ciclos o de las unidades de formación; escasa planificación de cronogramas; escaso conocimiento de para qué, cómo y qué se tiene que investigar sea para la teorización de la práctica o la experimentación de la teoría y; el escaso uso de 
la autoevaluación en el desempeño como practicantes.

Asimismo, desde el análisis documental se pudo identificar al proceso de inducción antes de ir a la práctica; la organización de la práctica preprofesional por niveles, ejes integradores, ejes vertebradores y resultados de aprendizaje de la práctica; la explicación general de las tareas que debe cumplir la pareja pedagógica practicante; el diseño, ejecución y evaluación de Ios PIENSA (Proyecto Integrador de Saberes) y; la evaluación prioriza lo formativo antes que la sumativo. De entre los aspectos negativos encontrados están: no se detalla funciones o tareas específicas que se deben ejecutar en cada ciclo o en las unidades de formación; la escasa orientación para aprender a utilizar métodos, técnicas e instrumentos de investigación acordes a la necesidad investigativa y; la escasa orientación para evaluar a los practicantes al término de cada unidad de formación.

\subsection{Segunda etapa: elaboración de la propuesta y pilotaje}

La elaboración de la propuesta se construye a partir de los resultados obtenidos en la primera etapa siguiendo la dimensión e indicadores. Se construye también por la revisión teórica de las categorías antes mencionadas y el análisis documental del Modelo de Práctica Preprofesional de la UNAE.

Una vez elaborada la propuesta inicial, se procedió a realizar el pilotaje de la misma. Este consistió en la presentación previa de la propuesta a un grupo de docentes y estudiantes de noveno ciclo de la UNAE, así como de tutores profesionales de la Escuela de Educación Básica Isaac A. Chico (lugar donde realizó la práctica preprofesional) considerando que son sujetos con más experiencia sobre práctica preprofesional y pareja pedagógica practicante.

Como resultado se obtuvo la aceptación de la dimensión y de los cincos indicadores establecidos. Es decir, ningún sujeto contradijo la propuesta. Además, se contó con importantes observaciones entre comentarios y 
recomendaciones enfocadas a la mejora de la propuesta de las acciones y criterios. En efecto, se obtiene la tabla de funciones mejorada después del pilotaje lista para ser presentada a la comunidad UNAE.

\subsection{Tercera etapa: presentación de la propuesta a estudiantes, docentes y directivos de la UNAE}

La propuesta mejorada se presenta a través de encuesta. Cabe indicar que tanto estudiantes como docentes pertenecen mayoritariamente a la carrera Educación Básica, mientras que los directivos son aquellos que regulan el funcionamiento de la práctica preprofesional en la UNAE.

De acuerdo a los resultados obtenidos de esta etapa, se puede decir que los mismos suceden igual que en la etapa anterior. Es decir, se contó con la aceptación de la dimensión y sus cinco indicadores por parte de los sujetos encuestados e importantes observaciones que permitieron seguir mejorando la propuesta. Por ejemplo, un estudiante comentó que las responsabilidades deben ser compartidas. Asumiendo dicho comentario los autores amplían al mismo en la fundamentación teórica y se menciona como criterio dentro de la tabla de funciones.

\subsection{Cuarta etapa: elaboración de la propuesta definitiva}

Es necesario recordar que la etapa tres permitió constatar la aceptación a la propuesta por parte de la comunidad UNAE. Por lo tanto, en esta última se elabora la propuesta definitiva considerando los componentes de la práctica preprofesional, los objetivos de las unidades de formación y el énfasis en el eje vertebrador para incorporar las tareas específicas de cada función.

Antes de abordar la tabla 1 de funciones, se presenta la propuesta de objetivos y énfasis del eje vertebrador para cada unidad de formación. Los mismos que están relacionados con los componentes de la práctica preprofesional en la UNAE. 
Tabla 1. Propuesta de objetivos y énfasis en el eje vertebrador para las unidades de formación.

\begin{tabular}{|l|l|l|}
\hline \multicolumn{3}{|c|}{ Unidad de formación } \\
\hline Básica & Objetivo de la unidad \\
\hline \multicolumn{3}{|c|}{ Trofesional } \\
\hline $\begin{array}{l}\text { Familiarización } \\
\text { de la profesión }\end{array}$ & $\begin{array}{l}\text { Consolidar el interés por la } \\
\text { profesión }\end{array}$ & $\begin{array}{l}\text { Transformar de practicante a } \\
\text { docente }\end{array}$ \\
\hline \multicolumn{3}{|c|}{ Eje vertebrador } \\
\hline Acompañar & Ayudar & Experimentar \\
\hline \multicolumn{3}{|c|}{ Núcleo problémico } \\
\hline Acompañar & Ayudar & Experimentar \\
\hline \multicolumn{3}{|c|}{ Eje integrador $8^{\circ}$} \\
\hline $\begin{array}{l}\text { De } 1^{\circ} \text { y } 2^{\circ} \text { ciclo } \\
\text { De } 3^{\circ} \text { y } 4^{\circ} \text { ciclo }\end{array}$ \\
\hline \multicolumn{3}{|c|}{ De $5^{\circ}, 6^{\circ}$ y $7^{\circ}$ ciclo } \\
\hline \multicolumn{3}{|c|}{ Cada ciclo cuenta con su propio eje } \\
\hline
\end{tabular}

Fuente: Los Autores (2019).

Un ejemplo para comprender tales propuestas es que: familiarizarse con la profesión, tomando como referencia a los ejes integradores, implica primero conocer las políticas públicas, contextos, modelos pedagógicos y modelos curriculares. Consolidar el interés por la profesión, tomando como ejemplo al eje integrador modelos curriculares, implica el conocimiento a profundidad de documentos como el PEI, PCl, entre otros. Transformarse de practicante a docente, siguiendo el mismo ejemplo, implica ya el diseño, ejecución, evaluación y reformulación de esos documentos.

Desde tales propuestas, se presenta el formato de la tabla 2, de funciones de la pareja pedagógica practicante que reúne las funciones, acciones, criterios y tareas específicas de cada función. La misma se estructura de la siguiente manera: 
Tabla 2. Formato de la tabla de funciones de la pareja pedagógica practicante.

\begin{tabular}{|c|c|c|c|c|c|}
\hline \multicolumn{6}{|c|}{ Funciones de la pareja pedagógica practicante } \\
\hline \multirow[b]{2}{*}{ 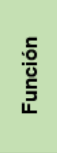 } & \multirow[b]{2}{*}{ Acciones } & \multirow[b]{2}{*}{$\begin{array}{l}\text { Criterios para el } \\
\text { reconocimiento } \\
\text { de la función }\end{array}$} & \multicolumn{3}{|c|}{ Tareas especificas según las unidades de formación y énfasis en el eje } \\
\hline & & & \begin{tabular}{l}
\multicolumn{1}{c|}{ Unidad Básica } \\
Objetivo: \\
Familiar con la profesión docente \\
Ejes vertebradores: \\
(acompañar, ayudar y experimentar)
\end{tabular} & \begin{tabular}{|l}
\multicolumn{1}{|c|}{ Unidad Profesional } \\
Objetivo: \\
Consolidar la profesión docente \\
Ejes vertebradores: \\
(acompañar, ayudar y experimentar)
\end{tabular} & \begin{tabular}{|l|}
\multicolumn{1}{|c|}{ Unidad de titulación } \\
Objetivo: \\
Transformar de practicante a docente \\
Ejes vertebradores: \\
(acompañar, ayudar y experimentar)
\end{tabular} \\
\hline \multirow{4}{*}{ 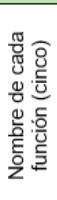 } & \multirow{4}{*}{ 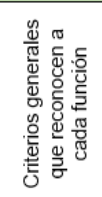 } & \multirow{4}{*}{ 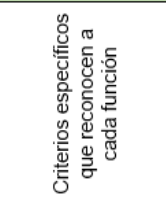 } & Tarea propia de la unidad & Tarea propia de la unidad & Tarea propia de la unidad \\
\hline & & & Tarea propia de ambas unidades & & Tarea propia de la unidad \\
\hline & & & Tarea propia de la unidad & \multicolumn{2}{|l|}{ Tarea propia de las tres unidades } \\
\hline & & & \multicolumn{3}{|l|}{ Tarea propia de las tres unidades } \\
\hline
\end{tabular}

Fuente: Los Autores (2019).

En efecto, la entrega de esta propuesta se considera un aporte a la construcción teórica del Modelo de Práctica Preprofesional de la UNAE. Es decir, un documento que permitirá a los practicantes conocer cuáles son sus funciones a cumplir en cada unidad de formación. Además, es un instrumento de trabajo que puede ser asumido por otras instituciones que adopten la práctica preprofesional como un componente básico en la formación inicial docente.

\section{Conclusiones}

El estudio de las funciones de la pareja pedagógica practicante constituye un objeto de investigación de significativa relevancia para nuestra universidad y una experiencia que puede ser valiosa y generalizable para otras instituciones con modelos de formación profesional donde la práctica constituya una de sus formas esenciales realización.

En el escenario de la práctica preprofesional se pudo observar el desempeño de la pareja y es donde se concretan las funciones propuestas como resultado de la investigación. Esta modalidad de formación docente concebida en la UNAE es crucial para fomentar el trabajo cooperativo, planificación, retroalimentación, cooperación, comunicación, organización, 
investigación, reflexión de la formación docente, entre otros aspectos que se viven cotidianamente en el transcurrir preparando a los practicantes a la labor profesional en función de competencias básicas y profesionales. Por otro lado, la formación en pareja pedagógica facilita la empatía, solidaridad, reciprocidad. La fundamentación permitió comprender la utilidad de la investigación acción participativa como metodología del paradigma socio-crítico.

La importancia de proponer las funciones de la pareja pedagógica practicante correspondiente a cada unidad de formación trasciende la labor propiamente docente para integrarse a la labor de formación profesional cuyo proceso se desarrolla de forma paulatina desde la unidad de formación básica a la unidad de profesionalización hasta la unidad de titulación. La propuesta proporcionó una tabla de funciones con sus respectivas acciones, criterios y tareas específicas descritas a partir de la intervención teórico-metodológico y el análisis de resultados de la encuesta aplicada. Esta tabla se acompaña de los objetivos de cada unidad de formación propuestos a partir de la experiencia como practicantes y están en sintonía con los ejes vertebradores, ejes integradores, núcleos problémicos, competencias básicas y profesionales y resultados de aprendizaje de la práctica preprofesional. En consecuencia, las tareas específicas y acciones se enmarcan en los siguientes indicadores: comunicativa, organizativa, ejecutiva, investigativa y evaluativa.

\section{Recomendaciones}

Para la ampliación de la propuesta, es necesario que se escudriñe las funciones desde las unidades de formación hasta las establecidas por ciclo. De tal manera que la pareja pedagógica practicante conozca en detalle cuáles son las funciones a las que debe responsabilizarse.

Asimismo, se cree necesario que esta propuesta se considere en las otras carreras de la UNAE para que los practicantes tengan una guía que les oriente su desempeño en la práctica preprofesional sea en cada ciclo o por 
unidades de formación. Además, dada la importancia del trabajo en pareja pedagógica practicante, se recomienda al Ministerio de Educación del Ecuador considerar esta modalidad en el trabajo docente y de prácticas preprofesionales.

\section{Referencias}

Brönstrup, C., \& Godoi, E., \& Ribeiro, A. (2007). Comunicación, lenguaje y comunicación organizacional. Signo y Pensamiento, XXVI(51), 2637, e-ISSN: 0120-4823. Recuperado de:

https://www.redalyc.org/articulo.oa?id=86005104

Carniato, J., Tulián, Y., \& Velázquez, S. (2015). La Pareja Pedagógica, Una Experiencia de Valor Formativo que Enriquece las Primeras Prácticas Docentes de los Alumnos de Profesorado en Ciencias de la Educación. $1^{\circ}$ Congreso Internacional sobre la Formación del Profesorado "Narración, Investigación y Reflexión sobre las prácticas". ISBN: 978-987-544-655-7. Buenos Aires, Argentina: Facultad de Humanidades de la Universidad Nacional de Mar del Plata.

González, N., Zerpa, M., Gutiérrez, D., \& Pirela, C. (2007). La investigación educativa en el hacer docente. Laurus, 13(23), 279-309, e-ISSN: 1315-883X. Recuperado de:

https://www.redalyc.org/articulo.oa?id=76102315

López, F. (2005). “Administración”, “Organización” (y "Empresa”): Un Intento de Acotación Semántica. Revista Universidad EAFIT, 41(137), 9-18, ISSN: 0120-341X. Medellín, Colombia: Universidad de EAFIT. Recuperado de: http://www.redalyc.org/pdf/215/21513702.pdf Matos, Y., \& Pasek, E. (2005). Planificación y ejecución de la investigación en equipo: un constructo. Revista de Artes y Humanidades UNICA, 6(14), 102-122, e-ISSN: 1317-102X. Recuperado de: 
Matos, Y., Cegarra, O., \& Rivera, C. (2017). La Praxis Docente desde la Formación Permanente. Revista Scientific, 2(4), 319-336, e-ISSN: 2542-2987. Recuperado de:

https://doi.org/10.29394/scientific.issn.2542-2987.2017.2.4.18.319-336 Mora, A. (2004). La evaluación educativa: Concepto, períodos y modelos. Revista Electrónica "Actualidades Investigativas en Educación", 4(2), 128, e-ISSN: 1409-4703. Recuperado de:

https://www.redalyc.org/articulo.oa?id=44740211

Porras, N. (2016). Acompañamiento pedagógico como estrategia para la transformación de la enseñanza de las matemáticas con los docentes de básica primaria de la Institución Educativa Manuela Beltrán. Trabajo final de maestría. Medellín, Colombia: Facultad de Ciencias de la Universidad Nacional de Colombia. Recuperado de: http://bdigital.unal.edu.co/51628/1/43277146.2016.pdf

RAE (2001). Investigar. 22. ${ }^{a}$ edición. Diccionario de la lengua española. Madrid, España: Real Academia Española. Recuperado de:

https://dle.rae.es/?w=investigar

Torres, M., \& Torres, C. (2005). Formas de participación en la evaluación. Educere, 9(31), 487-496 e-ISSN: 1319-4910. Recuperado de:

https://www.redalyc.org/articulo.oa?id=35603109

UNAE (2018a,b,c). Modelo de Práctica Preprofesional. Resolución-S0-005No-023-CG-UNAE-R-2018. Ecuador: Comisión Gestora de la Universidad Nacional de Educación. Recuperado de:

http://repositoriounae.unae.edu.ec/normativa/2 normativa comision g estora/normativa 2018/2.pdf

UNAE (2017a,b). Modelo Pedagógico de la Universidad Nacional de Educación del Ecuador. [Repositorio Digital]. Ecuador: Universidad Nacional de Educación del Ecuador. Recuperado de:

http://repositorio.unae.edu.ec/handle/56000/148 


\section{Juan Gabriel Jaramillo Jimbo}

e-mail: jaramillo271296@gmail.com

Nacido en Orianga, Ecuador, el 27 de abril del año 1997.

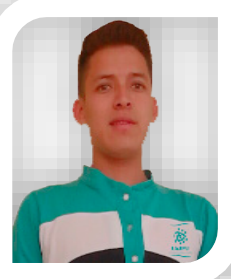

Soy licenciado en Ciencias de la Educación Básica con itinerario académico en Educación General Básica; Cuento con experiencia preprofesional en los niveles de Inicial, Básica y Superior; y profesional en subnivel Elemental; Dispongo de mucho interés, voluntad y compromiso para trabajar en instituciones educativas; Me caracteriza la puntualidad, flexibilidad, cooperación y responsabilidad en el desempeño profesional; Creo que una educación deseada es la que enseña a pensar, más que a memorizar; Me gusta la idea de trabajar en equipo, de ahí el motivo de mi trabajo de titulación. 


\section{Justina Elena Orovio Quintero}

e-mail: j_ecuador@hotmail.es

Nacida en Esmeraldas, Ecuador, el 6 de septiembre del

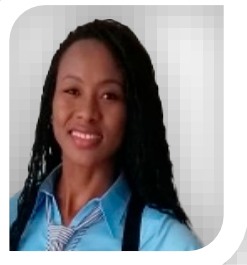
año 1990. Soy Licenciada en Ciencias de la Educación Básica con itinerario académico en General Básica; Cuento con experiencia en los niveles de Inicial y Básica; Con un gran interés de trabajar en instituciones educativas; Tengo un amplio historial relacionado con trabajo en equipo, cooperativo, liderazgo considerando la horizontalidad, entre otras características; de hecho, mi trabajo de titulación trata sobre el trabajo entre pares.

El contenido de este manuscrito se difunde bajo una Licencia de Creative Commons ReconocimientoNoComercial-Compartirlgual 4.0 Internacional 